\title{
Erythrocyte binding activity of Plasmodium vivax tryptophan rich antigens is inhibited by patients' antibodies
}

\author{
Rupesh K Tyagi, Yagya D Sharma \\ From Challenges in malaria research \\ Basel, Switzerland. 10-12 October 2012
}

\begin{abstract}
Background
Plasmodium vivax uses duffy antigen to invade the human erythrocytes but reports of duffy negative infection by $P$. vivax indicate that this parasite may also be using some other alternative receptors for the invasion [1]. Studies are therefore required to identify these additional parasite molecules which are involved in host-parasite interaction. Plasmodium vivax, contains a family of tryptophan rich antigens containing positionally conserved tryptophan residues. These antigens can induced significant cellular and humoral responses in human individuals $[2,3]$ and few showed interaction with erythrocytes.
\end{abstract}

\section{Materials and methods}

Recombinant Plasmodium vivax tryptophan-rich antigens were purified by Ni-NTA affinity chromatography. Heparinized blood was collected from healthy lab individuals to obtain human erythrocytes for binding assays, and from the microscopically confirmed P.vivax infected individuals for patients' sera. Rabbit antibodies against PvTRAgs used here were raised in the lab. Erythrocyte binding assays were performed by cell ELISA and Flow cytometry $[2,4]$.

\section{Results}

PvTRAgs binds to the human erythrocytes in a concentration dependent manner and not to the human lymphocytes. The binding was specific as competition with untagged proteins inhibits the erythrocyte binding to $50 \%$ at equimolar concentration. Antibodies raised against rabbits and produced by infected patients inhibit the binding of respective PvTRAg at different dilutions. PvTRAg38 and PvATRAg74 was sensitive to chymotrypsin only and not to the trypsin and neuraminidase while all other PvTRAgs were resistant to all these proteases.

\section{Conclusions}

Six out of fifteen PvTRAgs bind specifically to the human erythrocytes and were inhibited by rabbit anti PvTRAgs and also by the antibodies produced during natural course of $P$. vivax infection. There may be more than one RBC receptor for these six PvTRAgs where only PvTRAg38 and PvATRAg74 are sensitive to chymorypsin while others using those molecules which are other than glycophorin and sialoglycoproteins. Studies are in progress to investigate the respective $\mathrm{RBC}$ receptors for these PvTRAgs and their role in erythrocyte invasion process.

\section{Acknowledgements}

Department of Biotechnology, Government of India for financial support. Dr. Chetan Chitnis for providing DBP region II (PvRII) and Dr. S. S. Chauhan for his help in critical evaluation of the data.

Published: 15 October 2012

\section{References}

1. Mendes C, Dias F, Figueiredo J, Mora VG, Cano J, de Sousa B, do Rosario VE, Benito A, Berzosa P, Arez AP: Duffy negative antigen is no longer a barrier to Plasmodium vivax - molecular evidences from the African West Coast (Angola and Equatorial Guinea). PLoS Negl Trop Dis 2011, 5:e1192.

2. Alam MT, Bora H, Singh N, Sharma YD: High immunogenecity and erythrocyte-binding activity in the tryptophan-rich domain (TRD) of the 74-kDa Plasmodium vivax alanine-tryptophan-rich antigen (PvATRAg74). Vaccine 2008, 26:3787-3794.

3. Jalah R, Sarin R, Sud N, Alam MT, Parikh N, Das TK, Sharma YD: Identification, expression, localization and serological characterization of a tryptophan-rich antigen from the human malaria parasite Plasmodium vivax. Mol Biochem Parasitol 2005, 142:158-169.

4. Tran TM, Moreno A, Yazdani SS, Chitnis CE, Barnwell JW, Galinski MR: Detection of a Plasmodium vivax erythrocyte binding protein by flow cytometry. Cytometry A 2005, 63:59-66. 
doi:10.1186/1475-2875-11-S1-P98

Cite this article as: Tyagi and Sharma: Erythrocyte binding activity of Plasmodium vivax tryptophan rich antigens is inhibited by patients' antibodies. Malaria Journal 2012 11(Suppl 1):P98.

Submit your next manuscript to BioMed Central and take full advantage of:

- Convenient online submission

- Thorough peer review

- No space constraints or color figure charges

- Immediate publication on acceptance

- Inclusion in PubMed, CAS, Scopus and Google Scholar

- Research which is freely available for redistribution

Submit your manuscript at www.biomedcentral.com/submit 